\title{
ROBOT MAP BUILDING FROM SONAR SENSORS AND DSmT
}

\author{
Xinde LI, Xinhan HUANG and Min WANG
}

\begin{abstract}
Knowledge acquisition in map building presents characteristics of uncertainty and imprecision. Especially in the course of building grid map using sonar, this uncertainty is especially severe. Jean Dezert and Florentin Smarandache have recently proposed a new information fusion arithmetic (DSmT) whose greatest merit is to deal with uncertainty and conflict of information. In this paper, based on the arithmetic of DSmT, we can fuse information of different reliable degree for homogeneous or heterogeneous sensors. Then we established the belief model for sonar grid map, and constructed the generalized basic belief assignment function (gbbaf). Pioneer II mobile robot served as the experiment platform, and 3D Map was built based on DSmT online. At last, this paper established a firm foundation on studying dynamic unknown environment and multi-robots building map together and SLAM (simultaneous localization and mapping).
\end{abstract}

Keywords: Uncertainty, DSmT, grid map, information fusion, Mobile robot.

\section{Introduction}

The study on exploration of entirely unknown environment for intelligent mobile robots has being a popular and difficult subject for experts in robots field for a long time. Robots do not know the environment around themselves, that is, they have no experienced knowledge about the environment such as size, shape, layout of the environment, and also no signs such as beacons, landmarks, allowing them to determine their location about robot within the environment. Thus, the relation between self-localization and map building for mobile robot is like the chicken and egg problem [1,2]. This is because if the mobile robot builds the map of the environment, then it must know the determinate position of its own within the environment; At the same time, if the robot wants to know its own position, then it must have a referenced map of the environment. Though it is hard to answer this question, some intelligent sensors such as odometer, electronic compass, sonar detector, laser range finder and vision sensor are installed to the mobile robot as if a person has perceptive organs.

How to manage and utilize this perceptive information acquired by organs, it's a new subject in information fusion which will play an important role herein. As 
far as we know, experts have not yet given a unified expression. Just aiming to the practical field or system, proposed architecture of control such as hierarchical, concentrative, distributive and composite, and then according to the different integrated hierarchy, compared the validity of all kinds of classical (Probability) and intelligent (Fuzzy, Neural-Network (NN), Rough theory, Dempster-Shafer theory (DST), etc.) arithmetic. As far as mobile robot is concerned, the popular arithmetic of selflocalization in unknown environment relying on interoceptive sensors (odometer, electronic compass) and exteroceptive sensors (sonar detector, laser range finder and visual sensor) is Markov location [3] or Monte Carlo location [4]. The map of the environment is built by applying some arithmetic such as Probability theory, Fuzzy theory, Neutrosophic theory and NN. The information of environment can be expressed as grid map, geometrical feature or topological map, etc., where grid map is the most popular arithmetic expression [5, 6]. In this paper, DSmT mentioned here that has been proposed by Jean Dezert (French) and Florentin Smarandache (American) based on Bayesian theory and Dempster-Shafer theory [7] recently is a general, flexible and valid arithmetic of fusion. It is the largest advantage that it can deal with uncertain and imprecise information effectively, which supplies with a powerful tool to deal with uncertain information acquired by sonar detector in the course of building grid map. We present a new application of DSmT dealing with unreliable sensors based on the discounting method $[7,8,9,10]$.

\section{Fusion of unreliable sources with DSmT}

DSmT (Dezert-Smarandache Theory) is a new, general and flexible arithmetic of fusion, which can solve the fusion problem of different tiers including data-tier, featuretier and decision-tier, and even not only can dispose the static problem of fusion, but also can dispose the dynamic one. Especially, it has a prominent merit that it can deal with the uncertain and highly conflicting information [11, 12, 14].

\subsection{Simple review of DSmT}

1. Let $\Theta=\left\{\theta_{1}, \theta_{2}, \ldots, \theta_{n}\right\}$, here $\Theta$ is the frame of discernment, which includes $n$ finite focal elements $\theta_{i},(i=1, \ldots, n)$. Because the focal element is not precisely defined and separated, so that no refinement of $\Theta$ in a new larger set $\Theta_{\text {ref }}$ of disjoint elementary hypotheses is possible.

2. The hyper-power set $D^{\Theta}$ is defined as the set of all compositions built from elements of $\Theta$ with $\cup$ and $\cap\left(\Theta\right.$ generates $D^{\Theta}$ under operators $\cup$ and $\left.\cap\right)$ operators such that

(a) $\varnothing, \theta_{1}, \ldots, \theta_{n} \in D^{\Theta}$.

(b) If $A, B \in D^{\Theta}$, then $A \cap B$ and $A \cup B$ belong to $D^{\Theta}$. 
(c) No other elements belong to $D^{\Theta}$, except those obtained by using rules (a) or (b).

3. General belief and plausibility functions

Let $\Theta=\left\{\theta_{1}, \theta_{2}, \ldots, \theta_{n}\right\}$ be the general frame of discernment. For every evidential source $S$, let us define a set of map of $m():. D^{\Theta} \rightarrow[0,1]$ associated to it (abandoning Shafers model) by assuming here that the fuzzy/vague/relative nature of elements $\theta_{i}(i=1, \ldots, n)$ can be non-exclusive, as well as no refinement of $\Theta$ into a new finer exclusive frame of discernment $\Theta_{\text {ref }}$ is possible. The mapping $m($.$) is called a generalized basic belief assignment function if it satisfies$ [14]

$$
m(\varnothing)=0 \quad \text { and } \quad \sum_{A \in D^{\ominus}} m(A)=1
$$

$m(A)$ is called A's generalized basic belief assignment function (gbbaf). The general belief function and plausibility function are defined respectively in almost the same manner as within the DST, i.e.

$$
\begin{aligned}
& \operatorname{Bel}(A)=\sum_{B \in D^{\Theta}, B \subseteq A} m(B) \\
& \operatorname{Pl}(A)=\sum_{B \in D^{\Theta}, B \cap A \neq \emptyset} m(B)
\end{aligned}
$$

4. Classical (free) DSmT rule of combination

Let $\mathcal{M}^{f}(\Theta)$ be a free DSm model. The classical (free) DSm rule of combination, denoted (DSmC) for short, for $k \geq 2$ sources is given by $m_{\mathcal{M}^{f}(\Theta)}(\emptyset)=0$ and $\forall A \neq \emptyset, A \in D^{\Theta}$ by

$$
\begin{aligned}
m_{\mathcal{M}^{f}(\Theta)}(A) \triangleq & {\left[m_{1} \oplus \ldots \oplus m_{k}\right](A) } \\
= & \sum_{\substack{X_{1}, \ldots, X_{k} \in D^{\Theta} \\
X 1 \cap \ldots \cap X_{k}=A}} \prod_{i=1}^{k} m_{i}\left(X_{i}\right)
\end{aligned}
$$

\subsection{Fusion of unreliable sources}

\subsubsection{On the necessity of discounting sources}

In fact, sources of information are unreliable in real system due to the sources with different knowledge and experience. For example, from the point of view of mobile robots' sensors, the metrical precision and resolution with laser range finder are both higher than that with sonar detector. Even if they are the same sonar detector, then they 
have also different precision due to the making and other factors. Under these conditions, if we treat data of unreliable information sources as data of reliable sources to be fused, then the result is very unreliable and even reverse. Thus, unreliable resources must be considered, then DSmT based on the discounting method $[7,8,9,10]$ does well in dealing with unreliable sensors.

\subsubsection{Principle of discounting method}

Let's consider $k$ evidential sources of information $\left(S_{1}, S_{2}, \ldots, S_{k}\right)$; here we work out a uniform way in dealing with the homogeneous and heterogeneous information sources. So we get the discernment frame $\Theta=\left\{\theta_{1}, \theta_{2}, \ldots, \theta_{n}\right\}, m($.$) is the basic$ belief assignment, let $m_{i}():. D^{\Theta} \rightarrow[0,1]$ be a set of maps, and let $p_{i}$ represent reliable degree supported by $S_{i}, i=1,2, \ldots, k$, considering $\sum_{A \in D^{\Theta}} m_{i}(A)=1$, let $I_{t}=\theta_{1} \cup \theta_{2} \cup \ldots \cup \theta_{n}$ express the total ignorance, and then let $m_{i}^{g}\left(I_{t}\right)=$ $1-p_{i}+p_{i} m_{i}\left(I_{t}\right)$ represent the belief assignment of the total ignorance for global system after discounting, and then this is because of existing occurrence of malfunction, that is, $\sum_{A \in D^{\Theta}} m_{i}(A)=p_{i}$, we assign the quantity $1-p_{i}$ to the total ignorance again.

Thus, the rule of combination for DSmT based on discounting method with $k \geq 2$ evidential sources is given as the formula (3), i.e. the conjunctive consensus on the hyper-power set by $m_{\mathcal{M}^{f}(\Theta)}^{g}(\emptyset)=0$ and $\forall A \neq \emptyset, A \in D^{\Theta}$,

$$
\begin{aligned}
m_{\mathcal{M}^{f}(\Theta)}^{g}(A) \triangleq & {\left[m_{1}^{g} \oplus \ldots \oplus m_{k}^{g}\right](A) } \\
= & \sum_{\substack{X_{1}, \ldots, X_{k} \in D^{\Theta} \\
X 1 \cap \ldots \cap X_{k}=A}} \prod_{i=1}^{k} p_{i} m_{i}\left(X_{i}\right)
\end{aligned}
$$

\section{Modeling of sonar grid map building based on DSmT}

Pioneer II mobile robot is shown in Figure 1. Here we mainly discuss the sonar detector, whose working principle (shown as Figure 2) is: producing sheaves of cone-shaped wave, and detecting the objects by receiving the reflected wave. Due to the restriction of sonar physical characteristic, metrical data behaves out uncertainty as follows:

1. Beside its own error of making, the influence of external environment is also very great, for example, temperature, humidity, atmospheric pressure and so on.

2. Because the sound wave spreads outwards in the form of loudspeaker, and there exists a cone-shaped angle, we cannot know the true position of object detected among the fan-shaped area, with the enlargement of distance between sonar and it. 


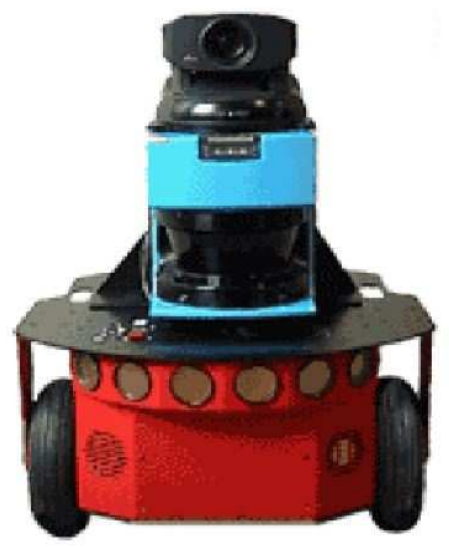

Figure 1: Pioneer II mobile robot

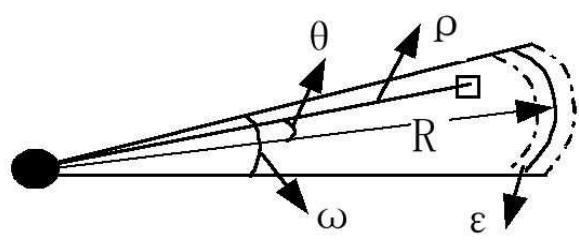

Figure 2: Sketch of the principle of sonar

3. The use of lots of sonar detectors will result in interference with each other. For example, when the $i$-th sonar gives out detecting wave towards an object of irregular shape, if the angle of incidence is too large, the sonar wave might be reflected out of the receiving range of the $i$-th sonar detector or also might be received by other sonar detectors.

4. Because sonar detectors utilize the reflection principle of sound wave, if the object absorbs very heavy sound wave, the sonar detector might be invalid.

Pointing to the characteristics of sonars measurement, we construct a model of uncertain information acquired from grid map using sonar based on DSmT. Here we suppose there are two focal elements in system, that is, $\Theta=\left\{\theta_{1}, \theta_{2}\right\}$, here $\theta_{1}$ means grid is empty, $\theta_{2}$ means grid is occupied, and then we can get its hyper-power set $D^{\Theta}=\left\{\emptyset, \theta_{1} \cap \theta_{2}, \theta_{1}, \theta_{2}, \theta_{1} \cup \theta_{2}\right\}$. Every grid in environment is scanned $k \geq 2$ times, each of which is viewed as a new source of evidence. Then we may define a set of map aiming to every source of evidence and construct the general basic belief assignment functions (gbbaf) as follows: 
- $m\left(\theta_{1}\right)$ is defined as the gbbaf for grid-unoccupied (empty);

- $m\left(\theta_{2}\right)$ is defined as the gbbaf for grid-occupied;

- $m\left(\theta_{1} \cap \theta_{2}\right)$ is defined as the gbbaf for holding grid-unoccupied and occupied simultaneous (conflict);

- $m\left(\theta_{1} \cup \theta_{2}\right)$ is defined as the gbbaf for grid-ignorance due to the restriction of knowledge and present experience (here referring to the gbbaf for these grids still not scanned presently ), it reflects the degree of ignorance of grid-unoccupied or occupied.

The gbbaf of a set of map $m():. D^{\Theta} \rightarrow[0,1]$ is constructed by authors according to formulas (5)-(8) based on sonar physical characteristics.

$$
\begin{aligned}
& m\left(\theta_{1}\right)=E(\rho) E(\theta)= \\
& \left\{\begin{array}{l}
\left(1-(\rho / R)^{2}\right) \lambda \text { if } \quad\left\{\begin{array}{l}
R_{\text {min }} \leq \rho \leq R \leq R_{\text {max }} \\
0 \leq \theta \leq \omega / 2
\end{array}\right. \\
0 \text { otherwise }
\end{array}\right. \\
& m\left(\theta_{2}\right)=O(\rho) O(\theta)= \\
& \left\{\begin{array}{l}
e^{-3 \rho_{v}(\rho-R)^{2}} \lambda \text { if }\left\{\begin{array}{l}
R_{\min } \leq \rho \leq R+\epsilon \leq R_{\max } \\
0 \leq \theta \leq \omega / 2
\end{array}\right. \\
0 \text { otherwise }
\end{array}\right. \\
& m\left(\theta_{1} \cap \theta_{2}\right)= \\
& \left\{\begin{array}{l}
{\left[1-\left[\frac{2(\rho-R+2 \epsilon)}{R}\right]^{2}\right] \lambda \quad \text { if } \quad\left\{\begin{array}{l}
R_{\min } \leq \rho \leq R \leq R_{\max } \\
0 \leq \theta \leq \omega / 2
\end{array}\right.} \\
0 \text { otherwise }
\end{array}\right. \\
& m\left(\theta_{1} \cup \theta_{2}\right)= \\
& \left\{\begin{array}{l}
\tanh (2(\rho-R)) \lambda \text { if }\left\{\begin{array}{l}
R \leq \rho \leq R_{\max } \\
0 \leq \theta \leq \omega / 2
\end{array}\right. \\
0 \text { otherwise }
\end{array}\right.
\end{aligned}
$$

where $\lambda=E(\theta)=O(\theta)$ is given by (see [5] for justification) 


$$
\lambda=\left\{\begin{array}{l}
1-(2 \theta / \omega)^{2} \\
0 \text { otherwise }
\end{array} \text { if } 0 \leq|\theta| \leq \omega / 2\right.
$$

and where, $\rho_{v}$ in formula (6) is defined as environment adjusting variable, that is, the fewer the object is in environment, the greater the variable $\rho_{v}$ is, and makes the function of $m\left(\theta_{2}\right)$ more sensitive. Here let $\rho_{v}$ be one. $E($.$) and O($.$) are expressed as the Effect$ Function of $\rho, \theta$ to grid's empty or occupancy. In order to assure the sum of all masses to be one, we must renormalize it. The analysis on the characteristics of gbbaf are shown on Figures 3-7, when $R=1.5$ meters.

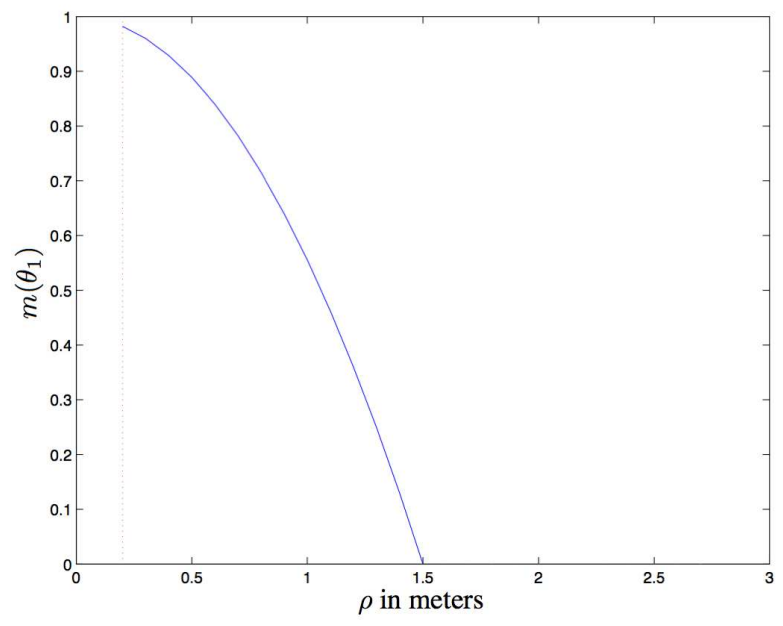

Figure 3: $m\left(\theta_{1}\right)$ as function of $\rho$ given by (5)

From Figure 3, one sees that $m\left(\theta_{1}\right)$ has a falling tendency with respect to the addition of distance between grid and sonar, and has the maximum at $R_{\min }$ and zero at $R$. From the point of view of the working principle of sonar, the more the distance between them approaches the measured value, the more that grid might be occupied. Thus the probability that the grid indicated is empty is very low, of course the gbbaf of grid-unoccupied is given low value.

From Figure 4, $m\left(\theta_{2}\right)$ takes on the distribution of Gaussian function with the addition of distance between them, has the maximum at $R$, which answers for the characteristic of sonar acquiring information.

From Figure 5, $m\left(\theta_{1} \cap \theta_{2}\right)$ takes on the distribution of parabola function with respect to the addition of distance between them. In fact, when $m\left(\theta_{1}\right)$ equals $m\left(\theta_{2}\right)$, $m\left(\theta_{1} \cap \theta_{2}\right)$ has the maximum there. But it is very difficult and unnecessary to find the point of intersection of the two functions. Generally, we let the position of $R-2 \epsilon$ replace the point of intersection. Experience indicates that its approximate value is more rational. 


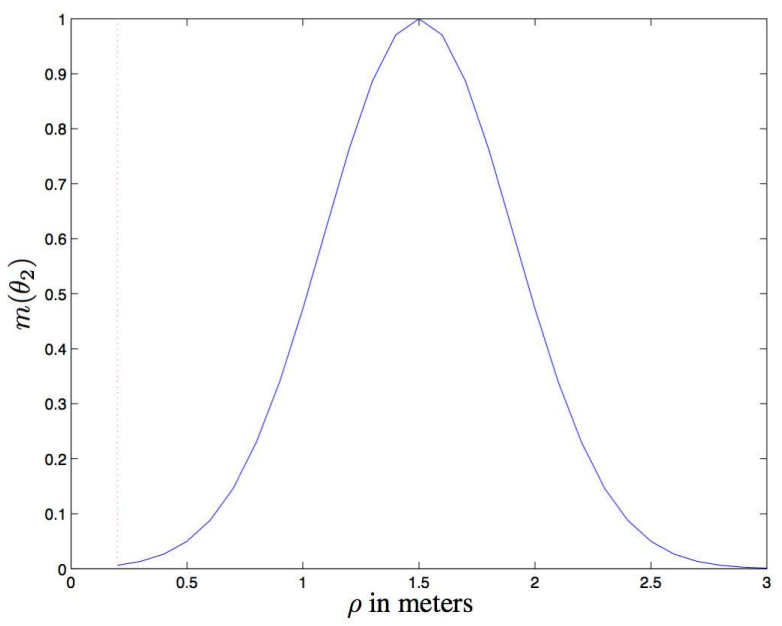

Figure 4: $m\left(\theta_{2}\right)$ as function of $\rho$ given by (6)

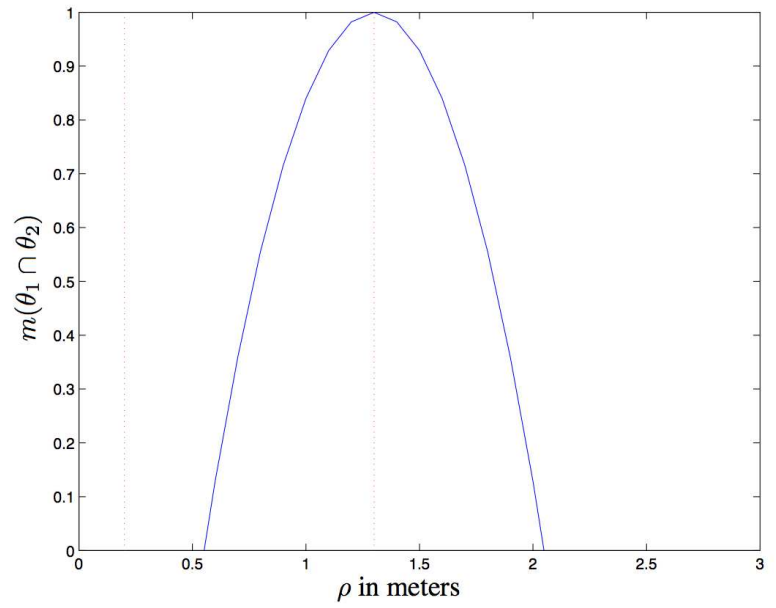

Figure 5: $m\left(\theta_{1} \cap \theta_{2}\right)$ as function of $\rho$ given by (7)

From Figure 6, $m\left(\theta_{1} \cup \theta_{2}\right)$ takes on the distribution of hyperbola function with respect to the addition of distance between them, and zero at $R$. This function reflects well the ignorance of grid information at $R \leq \rho \leq R_{\max }$.

The relation between $\theta$ and $\lambda$ is reflected in Figure 7, where the more the position of grid approaches central axis, the greater $\lambda$ becomes, that is, the greater the contribution to belief assignment is. Otherwise, the lower it is.

In short, the general basic belief assignment functions (gbbaf) entirely fit with the characteristic of sonar acquiring information. This supplies with a theoretic foundation 


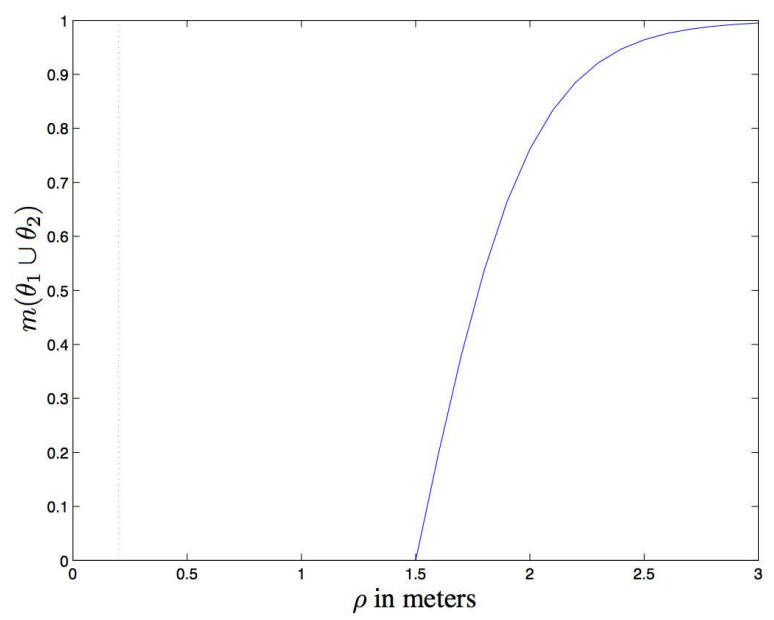

Figure 6: $m\left(\theta_{1} \cup \theta_{2}\right)$ as function of $\rho$ given by (8)

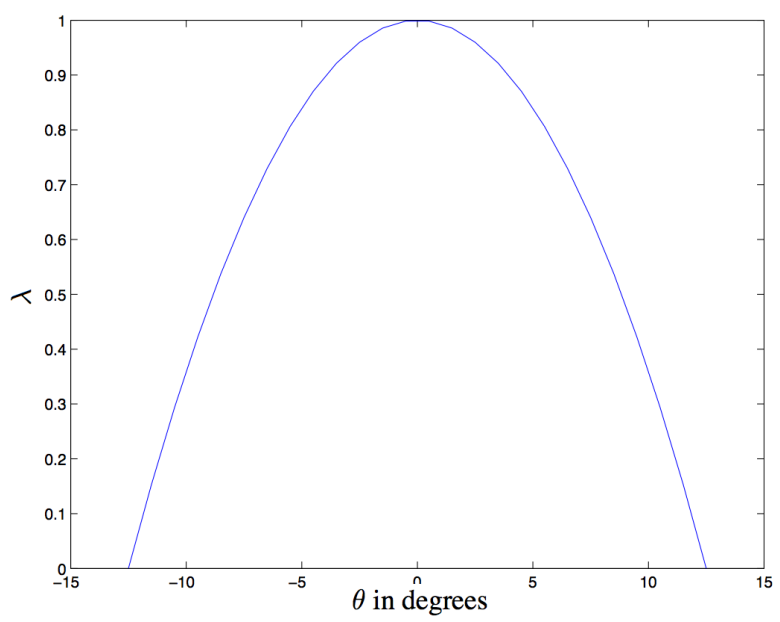

Figure 7: $\lambda$ as function of $\theta$ given by (9)

for dealing with uncertain information in grid map building. 


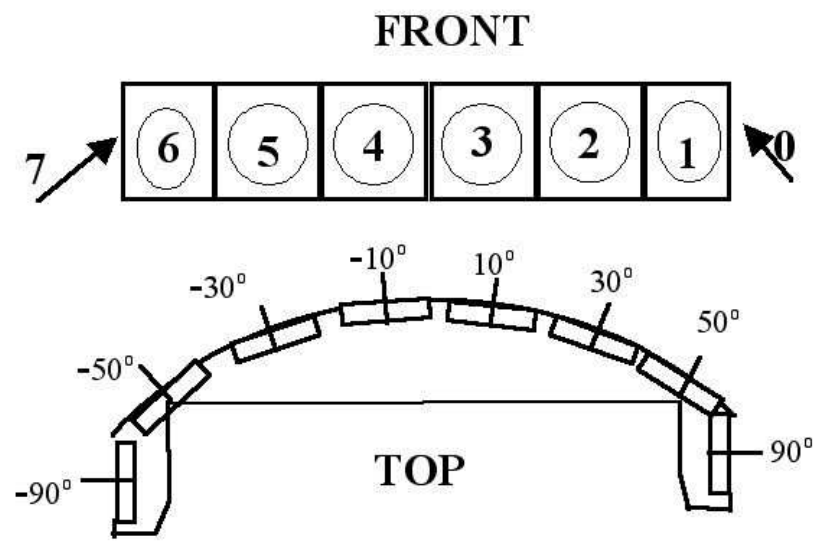

Figure 8: Sketch of the layout of sonars

\section{Experimental results}

\subsection{Fusion of Sonar information}

Pioneer II mobile robot has 16 Sonar detectors. Seen from Figure 8 , there are just 8 front sonar detectors shown, their distribution is asymmetrical. Flow chart of procedure of robot sonar map building based on DSmT is shown in Figure 13. Fusion steps are listed as follows:

We utilize the development tool of Visual $c++6.0$ on the platform of Linux, through the protocol of TCP/IP.

1. At the beginning of procedure, we proposed that all grids are fully occupied, that is, $\operatorname{Bel}\left(\theta_{2}\right)=1$, and mobile robot begins from origin of coordinate shown on Figure 9. Therefore, we may order the mobile robot to some spots. Of course, mobile robot's path planning and avoiding obstacles must be considered, but it is not an important point here. Robot may get the information of all sonar sensors at spot $(i, j)$. For simplified calculation, we apply the arithmetic of restricted spreading, which only computes the grid information in the fan-shaped area that each sonar can scan (shown on Figure 2)[13]. At the same time we also suppose that each sonar detector has different reliable degree, and the $i$-th sonar's one is $p_{i}(i=1, \ldots, 16)$. Here $p_{i}$ is acquired by experiment, in that, by judging his occurrence of malfunction. Of course, there is a rule: if the information from the different sonar is fused, the corresponding reliable degree ought to renormalize.

2. Utilizing the model of uncertainty belief established in the third part, through the formulations (5)-(9), gbbaf (such as $m\left(\theta_{1}\right), m\left(\theta_{2}\right), m\left(\theta_{1} \cap \theta_{2}\right)$ and $m\left(\theta_{1} \cup\right.$ 
$\left.\theta_{2}\right)$ ) are respectively computed. If sum of masses is not one, then we should renormalize it.

3. Judge whether the information of every grid scanned by all sonar sensors is new or not. If Yes, then goto step 1. Otherwise, goto next step.

4. Judge whether the grid is scanned repeatedly or not. If No, save the information of this grid. If Yes, goto step 5

5. Go on judging whether the fusion times is more than two times or not. If yes, and then stop fusing it. Otherwise, goto step 6.

6. Go on fusing it and at the same time, judge further. Whether the grids information is fused for the first time or not. If it is not the first time, then compute and update the Bel at last, then goto step 7. If it is the first time, goto step 8 .

7. Check whether Bel of all grids needs to be updated or not. If Yes, then goto step 9. Otherwise, goto step 1 .

8. Update the grid's original mass with the new mass after fusion. Then goto step 1 .

9. Stop and Exit the procedure at last.

\subsection{Analysis on the result of fusion}

We suppose the environment (size: $5 \mathrm{~m} \times 5 \mathrm{~m}$ ) partitioned in 2500 discrete even rectangular grids $(50 \times 50)$. Objects in rectangular grid map are sketched in Figure 9. Mobile robot wanders there, and gets 79 points of location for acquiring information (here to simplify, we only mark 3 key spots in Figure 9). Because the environment is smaller, moreover robot runs less time, the precision for self-localization of mobile robot is enough to realize the arithmetic. Of course, we had better consider the Markov or Monte Carlo position, especially when the environment is large and complex, because this can improve the quality of map building and can solve the chicken and egg puzzle. In fact, here we dont consider the optimal trajectory, if readers have some interest in this, they can try to find it. Presently we are also doing some research on it to economize the time and decrease the number of points of location. In order to improve the precision of fusion and weed out those under-proof data, let's suppose the mobile robot acquires information from three different directions for every point of location, and builds the map on-line according to the step of combination according to the section 4.1. 3D grid map is built based on DSmT, and shown on Figures 10-12 respectively when the numbers of location (n) is 23, 67, and 79.

The results are analyzed as follows: 


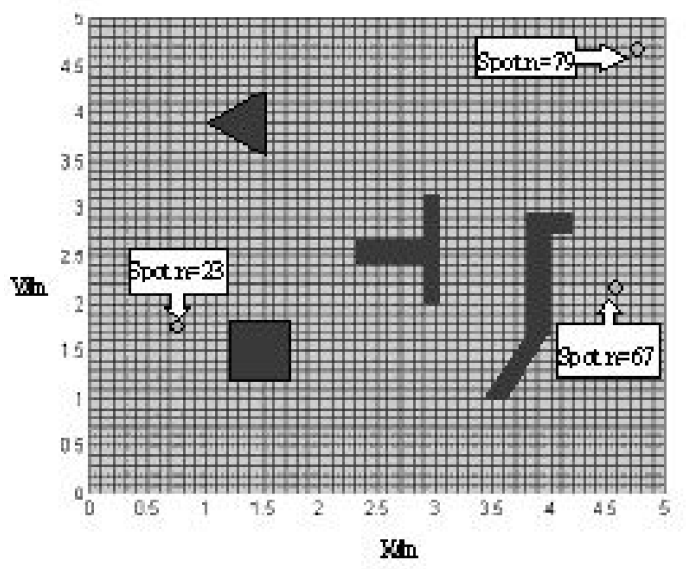

Figure 9: The grid map of original environment

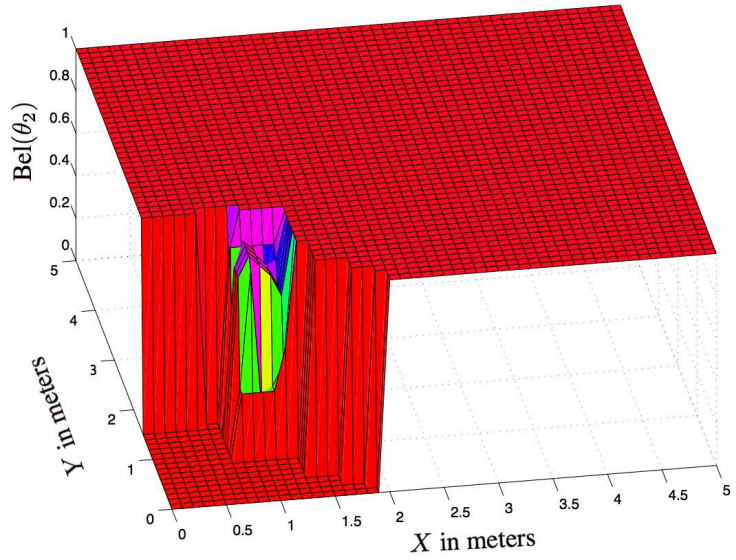

Figure 10: Estimation of grid map when $n=23$

a) Figures 10-12 show the process of building map online for Pioneer II mobile robot. In Figures 10-12, $Z$-axis shows the Belief of every grid occupied, zero shows the grid is empty fully, however, one shows this grid is fully occupied. Though the discerning rate is very high in this process with the increment of the complexity of the map, the discerning rate might decrease. However, the final discerning effect is very satisfying, which can be seen from the comparison between Figure 10 and Figure 12. This facilitates very much the development of human-computer interface of mobile robot exploring unknown, dangerous and sightless area. 


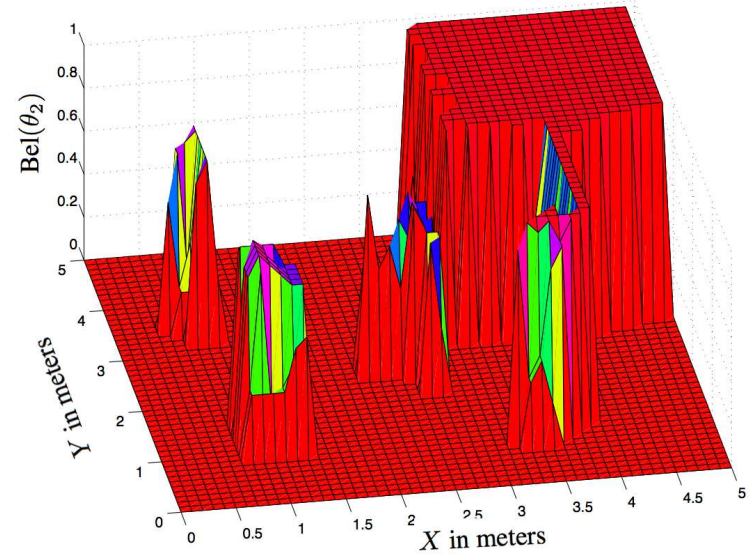

Figure 11: Estimation of grid map when $n=67$

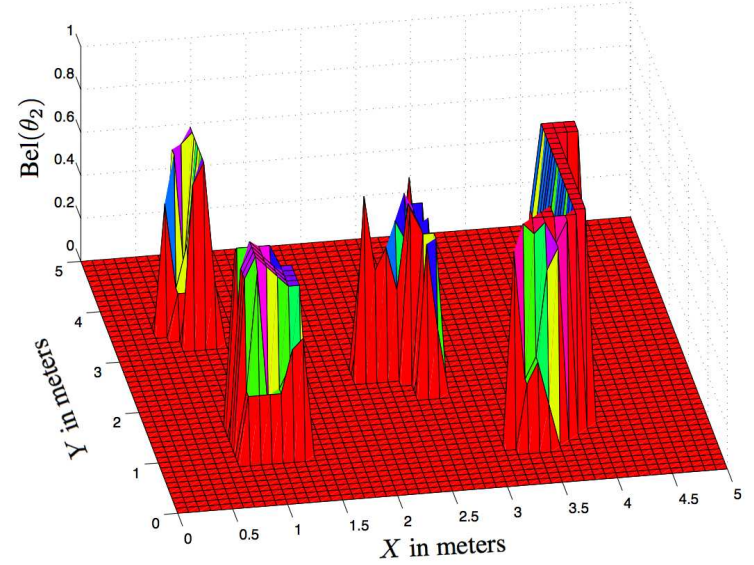

Figure 12: Estimation of grid map when $n=79$

b) Low coupling. Though there are many objects in grid map, but there occurs no phenomenon of the apparently severed, but actually connected. Thus, it supplies with a powerful evidence for self-localization, path planning and navigation of mobile robot.

c) High validity of calculation. The discounting approach as preprocessing for DSm fusion rule considering the restrained spreading arithmetic is adopted, and overcomes the shortcoming that the global grids in map must be reckoned once for sonar scanning every time, and improves the validity of calculation.

d) In this paper, we just apply classical model of DSmT to static environment, even 
if the environment's size is small, if the environment's size is very large and complex, then we must consider SLAM. While aiming to dynamic environment such as moving object and walking person therein, we must consider the hybrid model of DSmT [14].

\section{Conclusion}

In this paper, we applied DSmT based on the classical discounting approach to mobile robot's map building in a small environment. Then we established the belief model for sonar grid map, and constructed the generalized basic belief assignment function. Through the experiment, we get very better effect for robot's map building. At last, DSmT proved to be very valid. Of course, if the size of environment is very large and complex, or irregular or even a loop, the robot's position isn't neglected. Under this condition, we must consider robot's self-localization. In fact, we are doing some research in SLAM based on DSmT. We think, it is very important to improve the robustness and practicability of our arithmetic. In short, this study supplied with a shortcut for human-computer interface of mobile robot exploring unknown environment and established a firm foundation for the study of dynamic unknown environment and multi-robots' building map together.

\section{Acknowledgements}

We are grateful to Dr. Jean Dezert from ONERA/DTIM, Châtillon, France, for his comments and criticisms of this paper, and for his guidance, help and technical supports in DSmT. We also thank Professor Florentin Smarandache from Department of Mathematics, University of New Mexico, Gallup, USA, for his deep examination and correction of this paper.

\section{References}

[1] Montemerlo M., Thrun S., Simultaneous localization and mapping with unknown data association using Fast SLAM , Proceedings of the IEEE International Conference on Robotics and Automation, pp.1985-1991, 2003.

[2] Dasvison A. J., Murray D. W., Simultaneous localization and map building using active vision, IEEE Transactions on Pattern Analysis and Machine Intelligence, 24(7): pp. 865-880, 2002.

[3] Fox D., Burgard W., Thrun S., Active Markov localization for mobile robots, Robotics and Autonomous Systems, 25(12): pp. 195-207, 1998. 
[4] Thrun S., Fox D., Burgard W., Dellaert F., Robust Monte Carlo localization for mobile robots, Artificial Intelligence, Vol. 128, pp. 99-141, 2001.

[5] Elfes A., Moravec H., High resolution maps from wide angle sonar, IEEE Int. Conf. on Robotics and Automation, pp. 116-121, 1985.

[6] Elfes A., Sonar-based real-world mapping and navigation, IEEE Journal of Robotics Automat., Vol. 3, pp. 249-265, 1987.

[7] Shafer G., A Mathematical Theory of Evidence, Princeton University Press, Princeton, NJ, 1976.

[8] Dubois D., Prade H., Representation and combination of uncertainty with belief functions and possibility measures, Computational Intelligence, 4, pp. 244-264, 1988.

[9] Lefèvre E., Colot O., Vannoorenberghe P., Belief functions combination and conflict management, Information Fusion Journal, Elsevier Publisher, Vol. 3, No. 2, pp. 149-162, 2002.

[10] Smets Ph., Data Fusion in the Transferable Belief Model, Proc. of Fusion 2000 Intern. Conf. on Information Fusion, Paris, July 2000.

[11] Dezert J., Smarandache F., On the generation of hyper-power sets for the DSmT, Proceedings of the 6th Int. Conf. on Information Fusion, pp.1118-1125, FUSION 2003, Cairns, Queensland, Australia, 8-11 July 2003.

[12] Dezert J., Smarandache F., Partial ordering of hyper-power sets and matrix representation of belief functions within DSmT, Proceedings of the 6th Int. Conf. on Information Fusion, pp.1230-1238, FUSION 2003, Cairns, Queensland, Australia, 8-11 July 2003.

[13] Wang W. H., Map building by mobile robots in unknown environment, Ph. D. Thesis, Shanghai Jiao Tong University, 2003.

[14] Smarandache F., Dezert J. (Editors), Applications and Advances of DSmT for Information Fusion, American Research Press, Rehoboth, 2004,

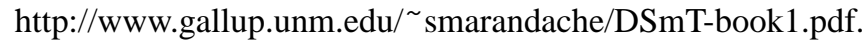


XINDE LI was born in Shandong province, China, on September 30,1975, He graduated from Shenyang institute of chemistry technology, Shenyang, China in 1997, received Master degree from Shandong University, Jinan, China, in 2003, presently was a Ph.D student in Department of Department of Control Science and Engineering of Huazhong University of Science and Technology (HUST), Wuhan, China since 2003. His main interests include information fusion, intelligent control,pattern recognition, robot' map building and localization, multi-robot system.

He is currently studying in Intelligent Control and Robotics Laboratory, Department of Control Science and Engineering, Huazhong University of Science and Technology, Wuhan 430074, China Tel: 027-87556242; Email:xdli825@yahoo.com.cn

XINXAN HUANG was born in Hubei province, China, on August 21, 1946. He graduated from Huazhong University of Science and Technology (HUST), Wuhan, China in 1969. He is Faculty member of the Department of Control Science and Engineering of HUST. He joined the Robotics Institute of Carnegie-Mellon University at Pittsburgh, USA as a visiting scholar from 1985 to 1986 and the Systems Engineering Division of Wales University at Cardiff, UK as a senior visiting scholar in 1996. He is currently a Professor and Head of the Intelligence and Control Engineering Division of HUST. The National Natural Science Foundation of China and the National High Technique Research Developing Plan award Professor Huang for his research projects from 1988 to 2005 . His research interests are the areas of robotics, sensing techniques, data fusion and intelligent control. He has more than 250 research publications to his credit. Professor Huang is a senior member of the Chinese Automation Society and Chairman of the Intelligent Robot Specialty Committee of the Chinese Artificial Intelligence Society.

MIN WANG was born in Wuhan, China, on July 27, 1954. She received BE and ME degrees from the Department of Automatic Control Engineering of Huazhong University of Science and Technology (HUST), Wuhan, China in 1982 and 1989 respectively. She is currently a Professor of the Department of Control Science and Engineering of HUST. The Chinese National Natural Science Foundation and the National High Technique Research Developing Plan award Professor Wang for her research projects from 1993 to 2005 . Her research interests include robotics, sensing techniques, neural networks and its applications. She has published more than 80 research papers in Journals and Proceedings. Professor Wang is a member of the Chinese Automation Society and Secretary-general of the Intelligent Robots Specialty Committee of the Chinese Artificial Intelligence Society. 


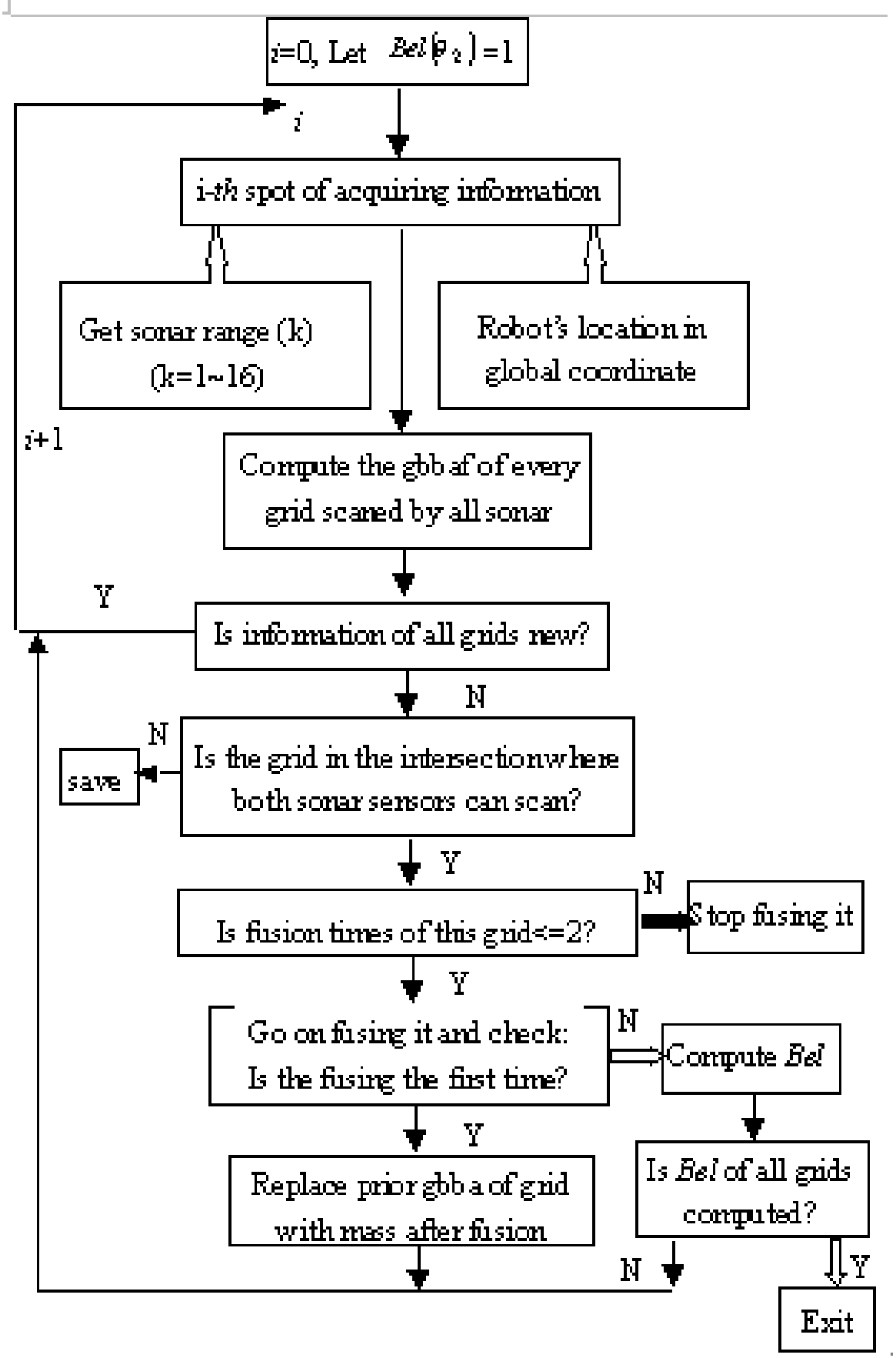

Figure 13: Flowchart of procedure of sonar map building based on DSmT 